\title{
Archéologie dans l'Europe contemporaine (projet ACE) : perspectives européennes sur la médiation au public et la profession archéologique
}

Kai Salas Rossenbach, Nathan Schlanger et Sonia Lévin

\section{(2) OpenEdition}

\section{Édition électronique}

URL : https://journals.openedition.org/archeopages/780

DOI : $10.4000 /$ archeopages.780

ISSN : 2269-9872

\section{Éditeur}

INRAP - Institut national de recherches archéologiques préventives

\section{Édition imprimée}

Date de publication : 1 octobre 2010

Pagination : 68-73

ISSN : 1622-8545

\section{Référence électronique}

Kai Salas Rossenbach, Nathan Schlanger et Sonia Lévin, « Archéologie dans l'Europe contemporaine (projet AcE) : perspectives européennes sur la médiation au public et la profession archéologique ", Archéopages [En ligne], Hors-série 2 | 2010, mis en ligne le 01 octobre 2010, consulté le 23 février 2023. URL : http://journals.openedition.org/archeopages/780 ; DOl : https://doi.org/10.4000/ archeopages.780 
des missions. Au cas par cas, on constate ici de fortes divergences qui ne tiennent le plus souvent qu'à une histoire particulière et au rôle de telle ou telle personnalité. Des conventions entre les instituts et les missions seraient souvent les bienvenues, y compris pour résoudre le difficile problème de la publication (pas forcément toujours sur papier) et de la diffusion des résultats. Il s'agit aussi d'articuler la recherche et la formation à la recherche, tout en évitant le concept ambigu et donc dangereux de « chantier-école» qui laisse, à juste titre, nos partenaires perplexes: comme si, pour la formation initiale de ses étudiants à l'archéologie, la France devait les envoyer s'exercer sur le patrimoine d'autres pays (le plus souvent extra-européens) alors qu'elle peut aussi bien les former en France à moindre coût. Bien évidemment les choses changent quand il s'agit de jeunes chercheurs engagés dans une thèse et qui ont, dès lors, un besoin indispensable de travail sur le terrain de leur sujet.

5 - Tout cela débouche sur des logiques de chaînes opératoires qui dépassent les clivages régionaux et qui devraient se mettre en place parallèlement à des réglementations coordonnées et cohérentes dans l'espace européen, ce qui n'est pas encore le cas. Cela permettrait des approches qui remettraient les territoires au centre de nos attentions. Territoires pris dans un sens de cohérence des écosystèmes, de l'histoire et des positionnements dans le monde d'aujourd'hui. L'archéologie est une discipline qui, par sa nature même, peut ambitionner de tenir le rôle de liant (sans impérialisme ni volonté de domination) dans une approche territoriale qui cesserait définitivement d'opposer le paysage au patrimoine tout en prenant en compte, dans le cadre du rassemblement des nécessaires compétences, toutes les facettes et toutes les potentialités d'un espace où ont vécu les hommes d'hier et où vivent les hommes d'aujourd'hui, afin de préparer un avenir plus serein pour les hommes de demain.

\section{Archéologie dans l'Europe contemporaine (projet ACE) : perspectives européennes sur la médiation au public et la profession archéologique}

\author{
Kai Salas Rossenbach \\ Nathan Schlanger \\ Sonia Lévin \\ Coordination du projet ACE/Inrap
}

0 cience du passé, l'archéologie est aussi une discipline éminemment contemporaine, ancrée de multiples façons dans le présent. Dès ses origines, avec une accélération depuis la deuxième moitié $\mathrm{du} \mathrm{xx}^{\mathrm{e}}$ siècle, et plus encore durant ces 20 ou 30 dernières années, l'archéologie ne se limite pas au stéréotype d'un savoir érudit, distant et souvent confidentiel, et moins encore à une chasse au trésor plus ou moins systématique pour alimenter collections et musées. Au-delà de ses volets scientifiques et culturels, l'archéologie comprend aussi, dans sa constitution et dans sa pratique, des dimensions sociales, économiques et politiques. À la fois ressource et enjeu, elle est aujourd'hui présente - et le plus souvent bienvenue dans nos campagnes et dans nos villes, dans nos paysages quotidiens et nos destinations lointaines, dans nos politiques culturelles, scientifiques et patrimoniales, dans nos stratégies d'aménagement $\mathrm{du}$ territoire et de son occupation citoyenne. Car, enfin, c'est bien dans le présent que l'archéologie œuvre pour l'avenir du passé. Interrogeons cette contemporanéité : qu'en est-il de sa dimension internationale, européenne? Les mouvements et les accords internationaux ont souvent eu, on le sait, un effet de levier sur les législations et les pratiques nationales - telle la convention Unesco sur le Patrimoine mondial (1972), ou encore les Cconventions du Conseil de l'Europe pour la protection du patrimoine archéologique (Malte, 1992) et du paysage (Florence, 200o). Peut-on mettre en évidence ces incidences et les comparer? Peut-on identifier des pistes et des écueils, cerner des différences structurelles et des ambitions communes, pays par pays, à l'échelle de notre continent? C'est bien ce défi que l'Inrap a choisi de relever, dans le cadre de sa politique de développement et de collaboration internationale et en vertu de sa stature, étant l'un des premiers instituts de recherche et de protection du patrimoine archéologique à l'échelle européenne, sinon mondiale.

L'Inrap s'est ainsi associé à une douzaine de partenaires européens pour concevoir un projet pluriannuel de coopération transnationale dans le cadre du programme «Culture » de la Commission européenne (2007-2012) intitulé «Archaeology in Contemporary Europe: professional practices and public outreach» (cf. www.ace-archaeology.eu). Avec l'Inrap comme chef de file et signataire, en partenariat avec l'Agence exécutive «Éducation, Audiovisuel et Culture » (EACEA) de la Commission Européenne, AcE est un réseau de collaborations qui réunit et met en œuvre une gamme d'expériences et d'expertises, afin d'analyser et de mieux faire connaître les enjeux et les pratiques de l'archéologie dans l'Europe contemporaine. Réunis dans un esprit de complémentarité et de partage, les partenaires du projet représentent une gamme d'institutions de différents statuts et tailles - des organismes de service public, des universités, des centres de recherche, des associations, etc. provenant de plusieurs pays d'Europe de l'Ouest, de la Méditerranée et d'Europe centrale . Dans le cadre de ce partenariat, et suivant les intérêts et les spécificités de chacun, le projet se développe autour de plusieurs thématiques qui font l'objet de recherches collectives, de présentations et de publications. Parmi les pistes ouvertes, qui sont ici brièvement exposées, l'une porte sur des actions de médiation et de diffusion au public, et l'autre concerne plus spécifiquement la thématique de la profession archéologique. 
Médiations et significations de l'archéologie.

Deux thématiques du projet ACE, abordées ensemble et de façon complémentaire, concernent « la signification du passé » et « la médiation aux publics ». L'intérêt que représente l'archéologie va au-delà de considérations érudites ou esthétiques, ou même simplement patrimoniales. L'archéologie apporte en effet une contribution unique à notre connaissance de l'histoire des sociétés anciennes, de leurs évolutions et de leurs interactions, de leurs cultures matérielles et de leurs rapports avec l'environnement. De ce fait, le passé porte en lui un potentiel de significations et de pertinence bien plus vaste, qu'il nous incombe, en tant que professionnels de son étude et de sa sauvegarde, de mettre en évidence et d'amener à la portée de tous. Dans cette perspective, le projet ACE cherche à comprendre comment cette signification est perçue, reconnue et évaluée par les différents publics, spectateurs et auditoires européens (archéologues amateurs, autorités locales, chargés de l'aménagement du territoire, visiteurs de musée, écoliers, télévisions et médias, etc.), et comment, de façon plus générale, l'archéologie est mise en œuvre comme moyen de construction identitaire, sociale et culturelle, en rapport, par exemple, à des phénomènes de migration et d'occupation de territoire. Étant donné ce potentiel, il est clair que la communication de l'archéologie et sa valorisation constituent un enjeu majeur de la discipline aujourd'hui. Dans le cadre du projet, il s'agit, d'une part, de faire partager compétences et méthodologies entre professionnels de la communication à l'échelle européenne, et, d'autre part (tenant compte des diverses idées reçues qui circulent sur les archéologues et leur profession), d'entreprendre des actions plus ciblées qui feront connaître quelques-unes des approches et des résultats obtenus à travers les autres thématiques du projet.

Les activités entreprises dans cette perspective comprennent des expositions, des publications et des festivals. Ainsi, le projet ACE a participé avec l'Inrap à la Ville européenne des sciences au Grand Palais en novembre 2008, dans le cadre de la présidence française de l'Union européenne. Avec ses partenaires de la Römisch-Germanische Kommission (Allemagne) et du Vlaams Instituut voor het Onroerend Erfgoed (Belgique), en charge d'ateliers interactifs, le projet a contribué à la conception d'un espace archéologique qui présentait, sur $160 \mathrm{~m}^{2}$, en une coupe stratigraphique de $3 \mathrm{~m}$ de haut [Fig.1], plusieurs facettes de l'archéologie contemporaine. Un des objectifs était de montrer aux quelque 40 ooo visiteurs que le passé, qu'il soit néolithique ou romain, ne pouvait être compris simplement dans le cadre de nos frontières nationales actuelles, et, parallèlement, que l'étude du passé faisait aujourd'hui appel à des compétences et des expertises réparties sur l'ensemble du continent et au-delà. De même, ACE a collaboré à la conception et l'édition de l'ouvrage collectif L'Europe archéologique. Un continent redécouvert par l'archéologie, paru en septembre 2009 aux éditions Gallimard/Inrap. Richement illustré et documenté, réunissant une gamme de spécialistes de différentes régions et périodes de l'archéologie européenne, cet ouvrage entend penser l'archéologie et l'histoire de l'Europe de manière globale, à l'échelle du continent, en montrant son unité et sa diversité, ses points communs et ses divergences. Toujours avec le soutien du projet ACE, l'Unité d'archéologie de la ville de Saint-Denis a entrepris sur l'îlot du Cygne, au cœur de la cité médiévale, une fouille archéologique qui a d'emblée inclus dans sa programmation des questions d'accès du public et de valorisation, et plus généralement de l'inscription du passé et de sa matérialité dans le territoire urbain et le tissu social actuel [Fig.2]. À l'autre bout de l'Europe, différentes enquêtes sur la signification du passé ont été présentées lors d'un colloque international organisé fin 2009 par l'Institut de préhistoire de l'Université Adam Mickiewicz de Poznań (UAM), suivi d'une publication intitulée Contemporary faces of the past. Enfin, le projet ACE apporte son soutien à l'association Kinéon et son festival international du Film archéologique. L'édition 2009 à Bruxelles était précédée d'un colloque international portant sur « How the media represent archaeology? What is at stake?». Publiées dans un ouvrage du même nom, les interventions à ce colloque ont porté sur les représentations de l'archéologue et de l'archéologie dans les médias (presse écrite, audiovisuelle ou littérature), afin de mieux cerner l'image de l'archéologue dans nos sociétés.

L'édition 2010 du festival, organisée dans le cadre du XII ${ }^{\mathrm{e}}$ festival Icronos à Bordeaux, a été, elle, précédée d'un colloque, toujours avec le soutien du projet ACE, sur " Archéologie et politique : quels liens, quels enjeux? » : les fouilles archéologiques à Silwan (Jérusalem), les bouddhas de Bamyan, le barrage de la Foz Coâ au Portugal ont notamment été évoqués.

Avec d'autres activités en cours et en préparation - une exposition photographique sur le métier d'archéologue qui sera montrée dans plusieurs villes européennes, un livre sur le métier d'archéologue pour les enfants de 3 à 5 ans, un colloque international sur « Les origines de l'Europe? Aspects culturels et sociaux des migrations médiévales »- le projet ACE concourt ainsi à explorer et à faire connaître les significations du passé et les réalités contemporaines de l'archéologie.

La profession archéologique : les enjeux contemporains. Les autres thématiques explorées par le projet ACE sont plus spécialisées et plus proches des enjeux opérationnels et des modes de fonctionnement de la discipline elle-même. Pour ce qui est des pratiques de l'archéologie, il s'agit d'entreprendre une évaluation comparative de la diversité des contextes et des structures dans lesquels elle se pratique. Les sous-thèmes abordés ici portent sur des questions de méthodologie de terrain et de traitement des données (notamment pour ce qui est des bases de données et des systèmes d'information, sous l'égide du partenaire 
ADS York, Royaume-Uni), des cadres administratifs et juridiques de l'archéologie au niveau national et international, et des pratiques de l'archéologie européenne sur la scène globale et spécifiquement dans les pays en voie de développement. Une première approche a mené à la publication, en 2008, de L'Archéologie préventive en Afrique: enjeux et perspectives, à la suite d'un état des lieux établi lors d'un colloque à Nouakchott sur la situation de l'archéologie préventive sur le continent africain aujourd'hui. Dans le cadre du projet ACE, cette question a fait l'objet d'enquêtes approfondies, pilotées par la faculté d'archéologie de l'université de Leyde (Pays-Bas), la RömischGermanische Kommission (Allemagne) et l'Inrap. Remontant aux fondements historiques de la discipline et au développement de la pratique archéologique en dehors des frontières nationales, les partenaires ACE ont cherché à identifier la place de leurs pays respectifs sur la carte contemporaine globale des programmes archéologiques, en rapport à des politiques de coopération et de développement international. Dans le cadre de cette thématique, une session intitulée «European Archaeology Abroad» s'est tenue à la réunion annuelle de l'Association européenne des archéologues (EAA) en 2010 à La Haye. Une publication des actes est en cours, ainsi qu'un ouvrage sur les dimensions culturelles et éthiques de l'activité archéologique à l'étranger aujourd'hui. Enfin, le projet ACE a co-organisé une session sur les effets de la crise économique globale sur l'archéologie lors de la réunion annuelle de l'EAA en 2009 à Riva del Garda, publiée depuis (en PDF, libre d'accès sur le site Internet d'ACE) sous le titre de Archaeology and the Global Economic Crisis: possible impacts, multiple solutions.

Le domaine où la crise $\mathrm{a}$ eu l'impact le plus fort est bien sûr celui de la profession. Étant données les conjonctures socio-économiques de réforme et de crise, et les diverses transformations récentes dans la nature et la pratique de l'archéologie et notamment de l'archéologie préventive, le projet ACE a reconnu d'emblée tout l'intérêt qu'il y avait à porter un regard plus européen et comparatif sur la question - d'où la thématique « la profession archéologique », pilotée par l'Inrap, le Laboratorio de Patrimonio de l'université Santiago de Compostela (Espagne) et le National Office of Cultural Heritage KÖH (Hongrie). En développant des questionnaires relayés par les partenaires, et en s'associant avec d'autres initiatives (dont celle du projet « Discovering the archaeologists of Europe») le projet entend explorer la profession archéologique tant dans ses aspects qualitatifs que quantitatifs, en reconnaissant au préalable toute l'importance qu'il faut accorder à la définition même de l'archéologue. La question de savoir qui est, ou qui compte, pour un archéologue professionnel, est en effet particulièrement complexe et variable, non seulement en fonction du temps et du lieu, mais aussi en rapport avec les finalités et les usages qui sont faits de cette définition. Il faut notamment garder en mémoire que la discipline archéologique se développe depuis le $\mathrm{xIX}^{\mathrm{e}}$ siècle avec un éventail particulièrement large de champs d'actions et d'expertises portant sur les dimensions matérielles du passé. L'histoire de l'art, la numismatique, l'architecture, la géologie, l'histoire, les sciences naturelles, l'anthropologie, l'ethnologie sont parmi les disciplines sollicitées, dont les praticiens collaborent de façon de plus en plus étroite et quotidienne avec les archéologues qui fouillent et étudient la culture matérielle. Au-delà de notions dépassées de sciences annexes ou auxiliaires, au-delà de dénominations hybrides tels archéozoologues ou paléo-botanistes, au-delà aussi de questions d'identité disciplinaire ou institutionnelle, il est évident que l'archéologie aujourd'hui met en œuvre un corps de techniques et de savoirs particulièrement diversifiés.

À cette diversité professionnelle académique s'ajoute celle que l'on peut qualifier de patrimoniale et qui découle, elle, non pas exclusivement de considérations scientifiques, mais aussi économiques et juridiques. Déjà au début du $\mathrm{xx}^{\mathrm{e}}$ siècle, lorsque les questions de droits aux fouilles, de découvertes fortuites et de contrôle par l'État commencent à se poser, émerge la nécessité de définir un personnel qualifié. Celui-ci devient indispensable avec la Convention pour la protection du patrimoine archéologique du Conseil de l'Europe, dite convention de Malte (1992), qui inclut la notion de personnel scientifiquement qualifié comme une des obligations des États signataires, et qui les encourage à développer des mécanismes de contrôle de qualité. C'est dans le contexte de cette mutation de l'archéologie que se pose frontalement la question de la légitimité d'obtention d'un permis pour entreprendre une activité archéologique (notamment de terrain, dans le cadre de l'archéologie préventive), sachant que des responsabilités patrimoniales et financières sont en jeu - et sachant aussi, malheureusement, que le monde des amateurs et des associations est de plus en plus écarté de la pratique archéologique. De ce fait, le critère le plus souvent (mais pas systématiquement) utilisé est celui du diplôme universitaire : qu'il soit adapté ou non, celui-ci apporte une certaine garantie des connaissances du domaine archéologique. Cependant, le diplôme tend parfois à se substituer à la somme d'expériences et de compétences que posséderaient des praticiens non diplômés, bien que professionnels avérés. Avec cet aspect d'expérience et de métier se pose la distinction entre « archéologue » et « technicien», ainsi que des différenciations et segmentations possibles de l'activité archéologique. Ces questions d'actualité, étant données les difficultés dans la gestion de l'activité archéologique selon différentes modalités d'emploi, mérite un regard comparatif à l'échelle européenne.

Alors que des textes européens définissant l'archéologie existent (par exemple le préambule de la convention de Malte), nous ne trouvons pas de définition européenne uniforme de la profession d'archéologue. Autrement dit, l'archéologie n'est pas régie par un système de désignation formelle dotée de licences et d'équivalences universitaires ou professionnelles, comme le sont en principe 

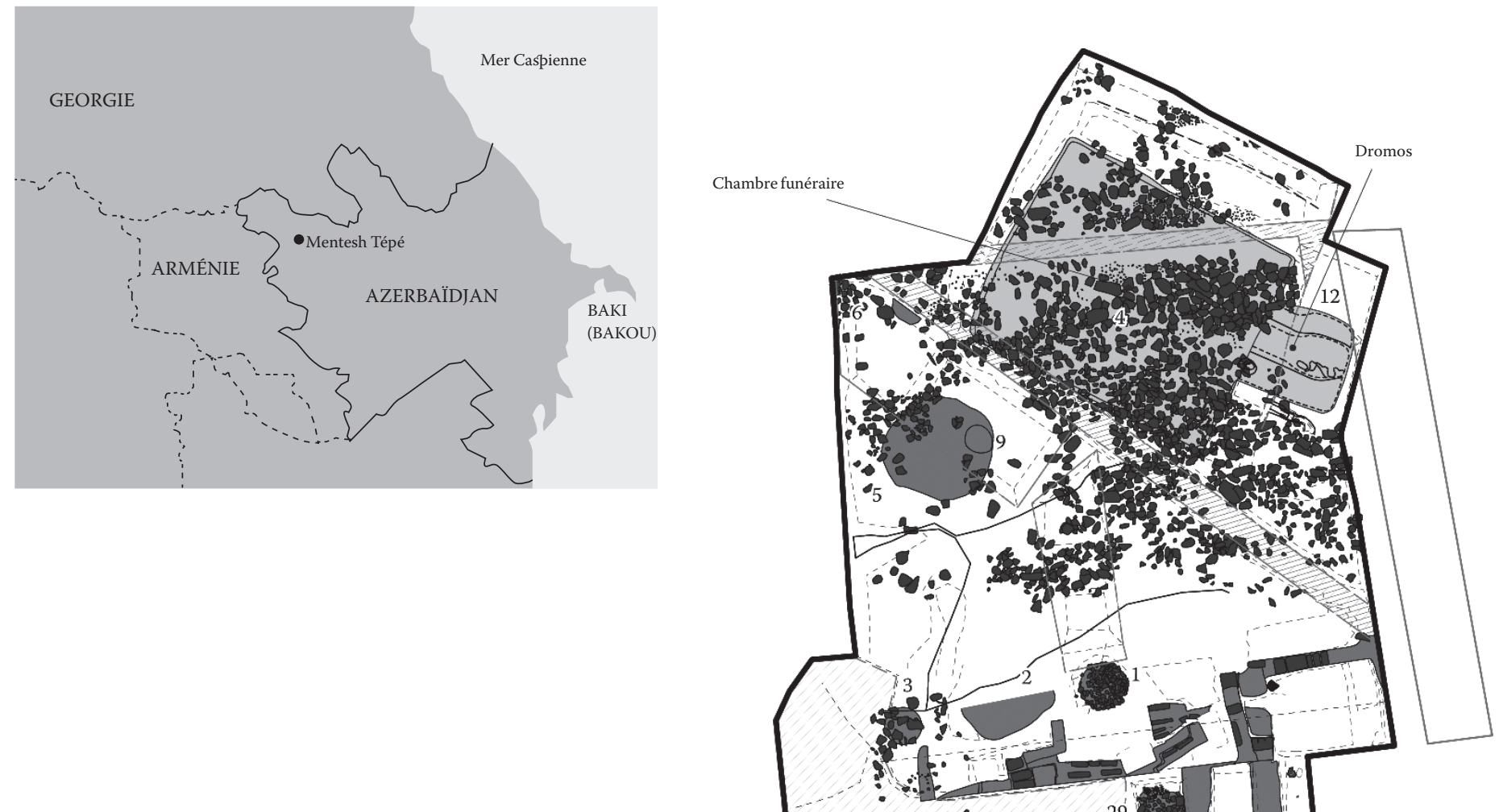

ก

[Fig. 1] Carte de localisation du site de Mentesh-Tépé en Azerbaïdjan.

[Fig.2] Mentesh-Tépé, plan général des vestiges.

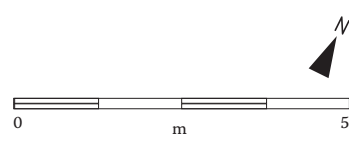

Structures Chalcolithiques.

Structures Kouro-Araxes. Sépulture collective ous kourgan.

$\square$ Sépulture individuelle.

Période indéterminé Perturbation contemporaine.

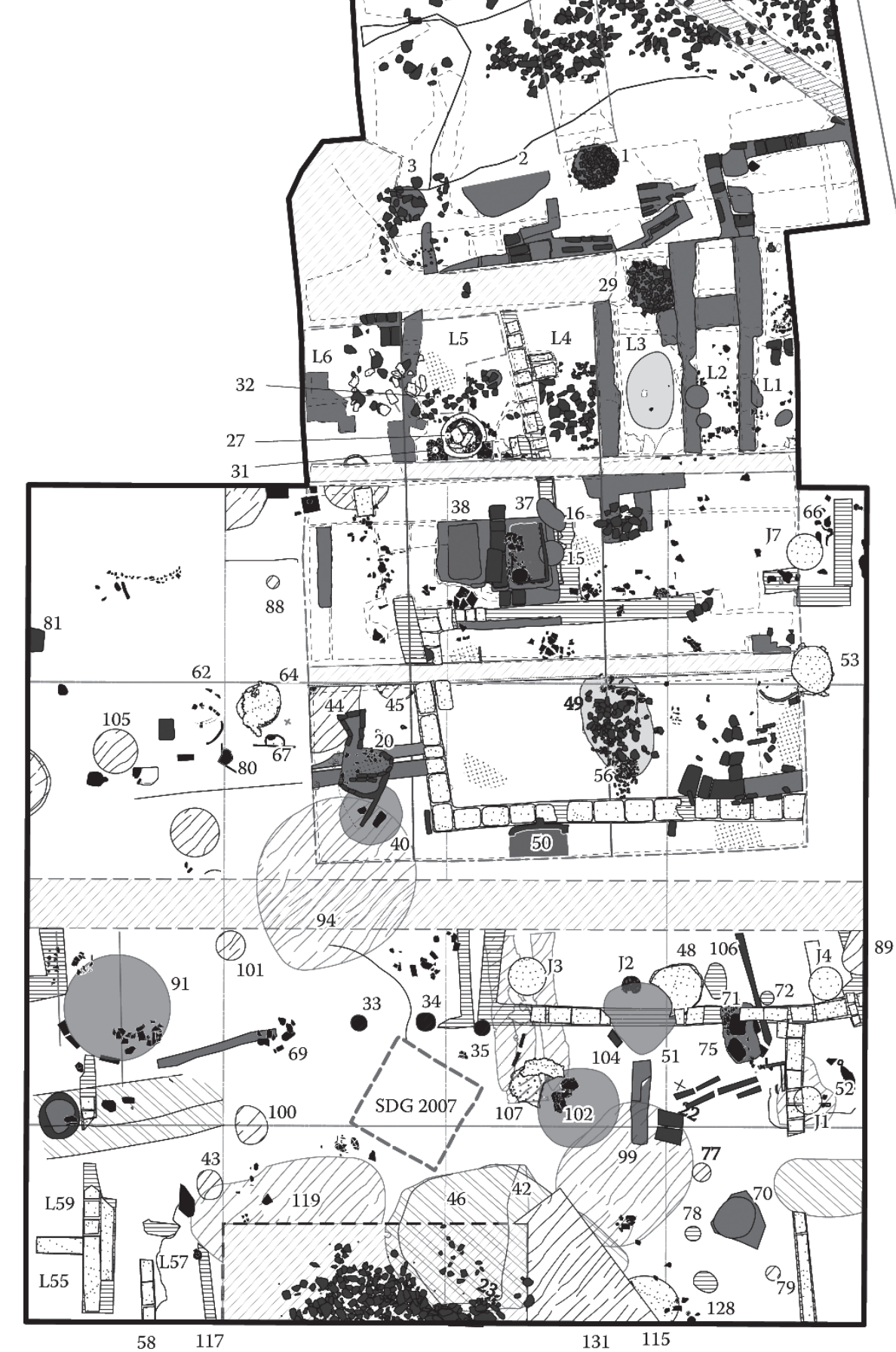


la médecine, la psychologie ou le droit. Dans certains pays européens, il n'existe aucune norme définissant l'archéologue - dans d'autres, une définition légale est en vigueur : avec l'essor de l'archéologie préventive, la tendance est clairement à la définition (et donc au contrôle) des conditions d'accès à la pratique de l'archéologie. Dans la plupart des cas, c'est le diplôme universitaire minimum qui joue un rôle déterminant.

Mis à part les cas du Royaume-Uni et de l'Irlande (qui ont à voir avec le système du common law et la commercialisation de l'activité archéologique) il faut noter que la relative cohérence du tableau [Fig.3] découle de l'harmonisation progressive des structures et des types de cursus universitaires effectuée depuis 1999 et le processus de Bologne : cette uniformisation n'est pas nécessairement synonyme de qualité ou d'égalité de formation à travers l'Europe. Au-delà du diplôme, les archéologues se définissent également par leurs expériences professionnelles. Cette notion d'expérience, associée principalement au terrain (le savoir-faire), est l'une des conditions mentionnées dans la plupart des pays pour l'attribution de permis, laissant l'autorité ad hoc juge de la nature et de la durée de l'expérience requise ou reconnue.

En France, si la reconnaissance des techniciens et des spécialistes est inscrite dans les textes, ils sont tous considérés comme des archéologues. Cette subtilité n'est pas stérile, elle montre au contraire une convergence des savoirs et des savoir-faire au sein de la discipline. La construction française de l'archéologie par le terrain autant que par le concept, associée à une tradition universitaire particulièrement non élitiste, a forgé une profession plus homogène qu'ailleurs. L'archéologue préventif français est le fruit de ce cadre. L'Inrap fait donc figure d'exception socioprofessionnelle dans le panorama de l'archéologie préventive en Europe.

Même s'il n'existe pas (et qu'il n'existera sans doute jamais) de définition unique et sans ambiguïté de l'archéologue professionnel en Europe, il devrait être possible d'arriver à une identification suffisamment ample et pertinente de critères applicables à l'échelle du continent. Ceci permettra de compléter et de renforcer l'approche qualitative de la profession archéologique par une approche quantitative. Une fois le nombre d'archéologues par pays défini de façon comparable - ainsi, techniciens compris, quelque 6800 en Angleterre, 4. 000 en Allemagne et 3500 en France (données 2008) - il devient possible de corréler ces chiffres avec une gamme d'indicateurs démographiques, géographiques, économiques et culturels, à l'échelle européenne : nombre d'archéologues par rapport à la population du pays, sa population active, sa population diplômée ; nombre d'archéologues par surface, par densité d'habitation, par réseau de transport; nombre d'archéologues par produit intérieur brut, par budgets d'aménagement du territoire et de BTP, par budgets dédiés à la culture et à la recherche, etc. À titre d'exemples, les deux encadrés suivants illustrent les premiers résultats des analyses.
Ces résultats quantitatifs pourront être corrélés à d'autres, telles les surfaces étudiées en diagnostic et en fouille, les surfaces « artificialisées » selon des données géo-satellitaires, mais aussi les budgets des opérateurs archéologiques, le nombre d'interventions, de rapports, de publications. Une fois rédigées et diffusées, ces données permettront, aux membres du projet ACE et à tous ceux qui le souhaitent, de mieux explorer les dimensions pratiques, scientifiques et patrimoniales de l'archéologie dans l'Europe contemporaine.

\section{L'archéologie préventive française, support et modèle pour la formation archéologique en Azerbaïdjan : l'exemple du site de Mentesh-Tépé}

\author{
Bertille Lyonnet \\ CNRS, UMR 7192 "Proche-Orient, Iran, Caucase : diversités \\ et continuités ", céramologue \\ Laure Pecqueur \\ Inrap, archéo-anthropologue \\ Pascal Raymond \\ Inrap, topographe \\ Anaïck Samzun \\ Inrap, UMR 7041 « Équipe Protohistoire européenne », archéologue
}

L 'Azerbaïdjan est sorti de la tutelle de l'Union soviétique après avoir acquis son indépendance en 1991. Ce pays est actuellement en plein développement, notamment grâce à l'exploitation des gisements pétrolifères de la mer Caspienne. L'intensité de sa reconstruction économique se conjugue avec sa volonté de préservation des vestiges archéologiques qui pourraient être détruits lors des travaux d'aménagement du territoire. Or, le pays est riche de sites archéologiques dont l'intérêt scientifique est majeur pour l'ensemble de la communauté scientifique. Il est donc nécessaire que son développement soit soutenu par des échanges qui lui apporteront les savoirs utiles à la valorisation de son patrimoine. La fermeture du pays aux évolutions techniques internationales, en particulier ces dernières décennies, n'a pas permis aux archéologues azerbaïdjanais d'acquérir la formation archéométrique et les compétences spécialisées de l'archéologie qui se sont largement développées en Occident à cette époque. Ces insuffisances de moyens techniques et de savoir-faire spécifiques expliquent le manque de rigueur des fouilles et des recherches afférentes conduites jusque-là. Les relevés précis de terrain, les analyses de l'industrie lithique ou osseuse, de la faune et de la flore, ou encore les examens anthropologiques sont encore très peu développés.

Historique de la mission et des recherches archéologiques en Azerbaïdjan. La mission « Boyuk Kesik», financée au départ par le ministère des Affaires étrangères et européennes, a été créée en 2006 à l'initiative de Bertille Lyonnet, en accord avec l'Institut d'archéologie et d'ethnologie de Bakou. Elle vise à mieux comprendre les relations 